recommended A-constant was used to calculate IOL power. The axial length adjustment with Wang-Koch modification was applied. The refractive value in the other eye determined the refractive aim in the operated eye.

In the Buckle group, 360 degrees encircling silicone band was inserted through four scleral tunnels at the beginning of surgery before phacoemulsification or inserting any trocars. The surgeries were done by two groups of surgeons according to their surgical preference, the first group adopted vitrectomy combined with scleral buckle and the second group adopted vitrectomy with retinectomy. Baseline characteristics of both groups were not statistically different which indicate that both groups were similar without any bias towards any of the two groups.

Heavy Silicon Oil Study which compares heavy and standard silicone oil (SO) in patients with inferior PVR failed to demonstrate superiority of a heavy tamponade [3]. Moreover, several complications have been associated with heavy SO surgery, such as prolonged intraocular inflammation and intraocular pressure increase, probably due to the early emulsification of heavy SO [4]. That is why we preferred to use $\mathrm{SO}(5000 \mathrm{cs})$ as a tamponading agent which has the least rate of emulsification [5].

The mean postoperative IOP was significantly higher in the Buckle group throughout the whole follow-up period. This may be due to impaired venous drainage from the vortex veins, leading to congestion of the ciliary body. The edematous ciliary body is displaced anteriorly, shifting the lens-iris diaphragm forward and resulting in narrowing of the angle [6]. Visual acuity was better at first month in the Buckle group, but this difference disappeared throughout the remaining followup period, achieving the same functional outcome.
The number of patients prevented subgroup analysis for the grades of PVR. Performing a prospective larger study for better statistical analysis will be a great idea. Once again, we would like to thank Awasthi et al. for sharing their comments.

\section{Compliance with ethical standards}

Conflict of interest The authors declare that they have no conflict of interest.

\section{References}

1. Awasthi U, Grover R, Videkar C, Varshney A. Comment on "Vitrectomy with scleral buckling versus with inferior retinectomy in treating primary rhegmatogenous retinal detachment with PVR and inferior breaks". Eye. 2018; epub ahead of print 18 December 2018.

2. Rahman R, Bong CX, Stephenson J. Accuracy of intraocular lens power estimation in eyes having phacovitrectomy for rhegmatogenous retinal detachment. Retina. 2014;34:1415-20.

3. Joussen AM, Rizzo S, Kirchhof B, Schrage N, Li X, Lente C, et al. Heavy silicone oil versus standard silicone oil in as vitreous tamponade in inferior PVR (HSO Study): interim analysis. Acta Ophthalmol. 2011;89:e483-9.

4. Theelen T, Tilanus MAD, Klevering BJ. Intraocular inflammation following endotamponade with high-density silicone oil. Graefes Arch Clin Exp Ophthalmol. 2004;242:617-20.

5. Zafar S, Shakir M, Mahmood SA, Amin S, Iqbal Z. Comparison of 1000-Centistoke versus 5000-Centistoke Silicone Oil in complex retinal detachment surgery. J Coll Physicians Surg Pak. 2016;26:36-40.

6. Gedde SJ. Management of glaucoma after retinal detachment surgery. Curr Opin Ophthalmol. 2002;13:103-9.

\title{
Smartphone adaptor use for nasal endoscopy
}

\author{
Jerome How Ing $\mathrm{Ha}^{1} \cdot$ Suresh Reddy Sagili ${ }^{1}$
}

Received: 11 December 2018 / Accepted: 19 December 2018 / Published online: 16 January 2019

(c) The Royal College of Ophthalmologists 2019

Supplementary information The online version of this article (https:// doi.org/10.1038/s41433-018-0334-6) contains supplementary material, which is available to authorized users.

Jerome How Ing Ha

dr.jeromeha@gmail.com

1 Department of Ophthalmology, Royal Shrewsbury Hospital, Mytton Oak Road, SY3 8XQ Shrewsbury, UK
Nasal endoscopic examination is an important part of the preoperative assessment in patients presenting with nasolacrimal duct obstruction, particularly when planning endoscopic dacryocystorhinostomy (DCR). Deviated nasal septum can impede the access to the middle meatus and identifying this preoperatively facilitates surgical planning for simultaneous septoplasty where indicated. Other pathologies such as synechia, nasal polyps and chronic 


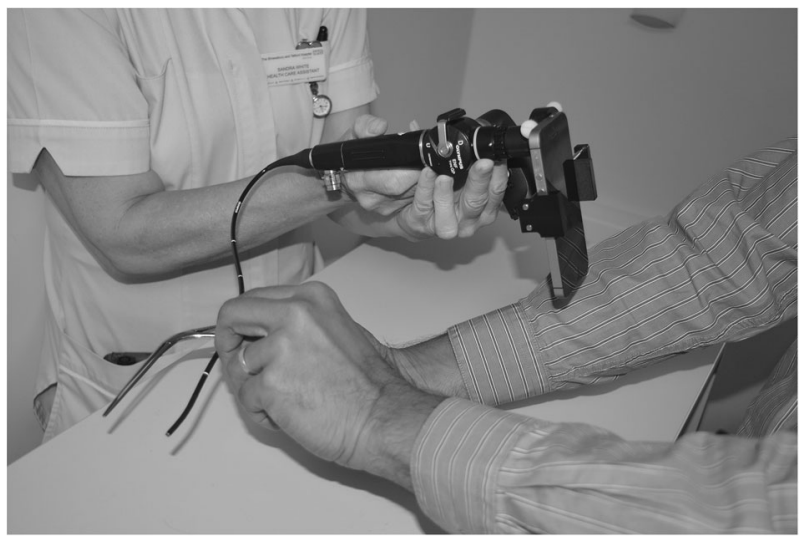

Fig. 1 Smartphone adaptor for nasal endoscopy set up

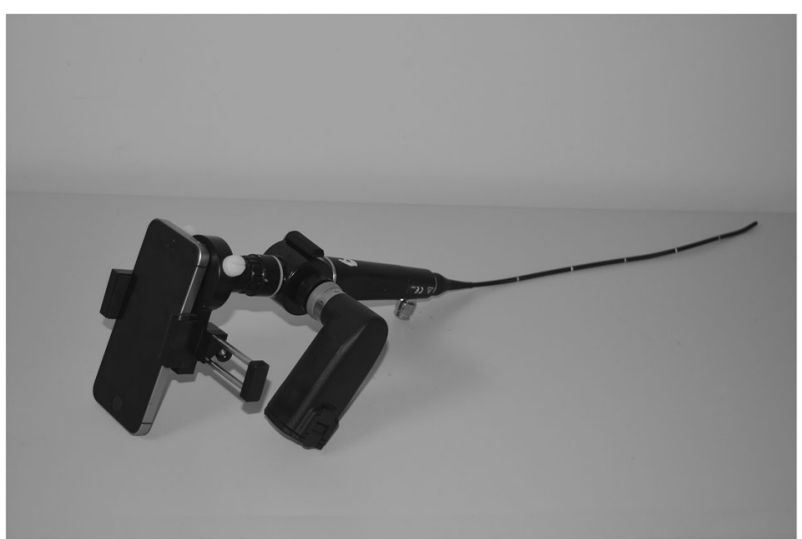

Fig. 2 Smartphone adaptor with the flexible nasal endoscope

rhino-sinusitis can also be identified during the nasal endoscopic examination preoperatively.

We routinely use a flexible endoscope for nasal examination in the clinic for preoperative assessment. A flexible endoscope is easier to use than a rigid endoscope [1] and is well tolerated without the need of topical nasal anaesthesia or decongestant. However, only one clinician can view through the eyepiece of the endoscope at any one time and it is not possible to demonstrate or teach simultaneously.

Using a smartphone adaptor enables live image display on the screen of the smartphone, which can be viewed by more than one clinician at a time [2].
It also allows the use of a flexible endoscope for removing silicone stents postoperatively, as the surgeon can use one hand to hold the forceps and the other to hold the tip of the flexible endoscope (Fig. 1). There is no need to hold the eyepiece of the endoscope, as this is attached to the smartphone and is held by an assistant (Fig. 1).

The smartphone adaptor (RVA Smart-Clamp) shown in figure 2 costs less than $£ 115.00$ and fits most of themodern smartphones (Fig. 2). This adaptor can be used with a flexible or rigid endoscope with a $31.75 \mathrm{~mm}$ eyepiece. There are several varieties of smartphone adaptors available in the market and we do not have any financial interest in any of the adaptors. Smartphone adaptor is significantly cheaper and a less cumbersome alternative than a camera with monitor attachment required for displaying the image.

We use a smartphone adaptor for preoperative and postoperative examination in patients undergoing DCR (see Supplementary video) and find it very useful in training junior doctors/fellows in the oculoplastic clinic. Attaching the smartphone adaptor to the flexible endoscope frees a hand of the clinician to allow removal of silicone stents postoperatively (see Supplementary video). We find this particularly useful, as flexible endoscope is more comfortable for patients compared to a rigid endoscope and can be used without the need of topical nasal anaesthesia or decongestant.

\section{Compliance with ethical standards}

Conflict of interest The authors declare that they have no conflict of interest.

Publisher's note: Springer Nature remains neutral with regard to jurisdictional claims in published maps and institutional affiliations.

\section{References}

1. Mohr RM. Endoscopy and foreign body removal. In: Paparella MM, Shumrick DA, Gluckman JL, Meyerhoff WL, editors. Otolaryngology. 3rd ed. Philadelphia: W.B. Saunders; 1991. p. 2399-425.

2. Wu CJ1, Wu SY, Chen PC, Lin YS. An innovative smartphonebased otorhinoendoscope and its application in mobile health and teleotolaryngology. J Med Internet Res. 2014;16:e71. 